\title{
The Research of Digital Training System for Sports
}

\author{
$\mathrm{Ni} \mathrm{ZHUO}^{1, \mathrm{a}}$ \\ ${ }^{1}$ Guangxi Technical College of Machinery and electricity, Nanning 530007 ,China \\ azhuoni1211@126.com
}

Keywords: Digital Training System; Sports Training; Petri Net; Sports Motion

\begin{abstract}
With the development of science and technology, science and technology of competitive has increasingly important role in promoting the development of undertakings of physical culture and sports. Give full play to the supporting role of science and technology, promote the development of undertakings of physical culture and sports in the new century is a major responsibility of the tech world, scientific training methods and means is an important and effective way to improve performance. This paper, through the analysis of physical connection principle from human skeleton model point based on the technology of digital computer, it is the combination of mining association rules between sports skill motion characteristics and model characteristics of data to establish physical skills of regular physical activity, to study physical training of digital system, so as to shorten the development cycle. Training, improve the athlete's level, provides a scientific research method.
\end{abstract}

\section{Introduction}

The development and application of modern science and technology changes with each passing day, the sports field, is no exception. In the process of the training of the athletes, from sports technical analysis, the whole body ability, training process, until the field of psychological preparation, the whole process is inseparable from the comprehensive science and technology, especially electronic information technology and equipment [1]. In the future, digital sports training will be in the competitive sports gradually revealed its unique charm. Human movement analysis of computer software is a hot issue in the field of computer software analysis, it has important application value in the field of sports analysis. At present, many research institutions at home and abroad are all in the related research work, committed to the development of track and field, boxing, skiing, table tennis and other sports analysis system. The use of these systems can greatly convenient and the training of the athletes, the athletes from different point of view in the process of training professionals for the execution of the details. When using this system, often need more practical athlete to complete the action with the corresponding standard action, the difference between athletes in order to evaluate the effect of the complete data and video is the athlete movement of the main form of storage, which requires the training video and professional athletes to compare.

\section{The human skeleton model}

Human joint angle data can be derived from the motion capture system or depth of processing camera Microsoft Kinect data. The key is how to construct a 3D human body model after data. The human skeleton model is the foundation of construction of the human body model. Since the main key point of three-dimensional position information contained, so can better represent the information of human body. Abstract the human skeleton model as shown in Figure 1, contains a total of 23 key points, at the root node of the waist. Because the motion capture data is based on the coordinates of the world coordinate system, we first make every action coordination root node is the origin of the world coordinate system, in order to make the action sequence data has nothing to do with the absolute position of the body [2]. 


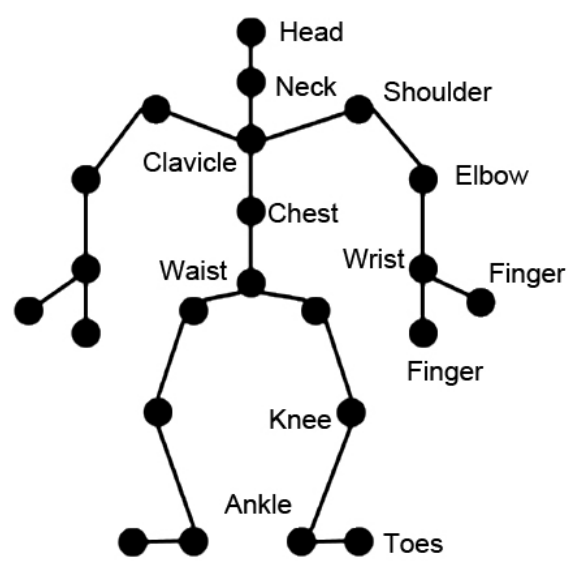

Figure1. 3D human skeleton model

At the same time, the body also is $\mathrm{Z}$ direction along the axis of the initial position, initial orientation and human data for independent. At the same time, we will body frame length and different people the same frame size, so that the operation of body size data independent.

Coordinate 23 joint points can be a unified standard, the data dimension effectively 67D (including the height information, root node section 0 ). We will also skeleton is decomposed into a series of parts, each part is a single key combinations or point. Node classification contains semantic information, let us identify and extract key part of more effective action.

\section{Sports skilled motion design of digital training system for sports}

Petrie network can be vivid, in-depth description of the processes of discrete event dynamic systems, the relationship between the accurate description of the sequence of events, concurrency and conflict. Petrie network applications to the field of sports, place and transition must be unique significance. Here, on behalf of the state of technology at the time of the place. Technology transition course of action. The relationship between the various technical actions or status of the place, the transition period director arc [3].

Combination of sports activities, we will base technology including elements of motion is designed as a place / transition net by Petrie, a loop (T2, T3, T4) scheduling technology complete elements, as shown in figure 2. Petrie network model clearly reflected the movement process technology, the relations between the various motion between, and the conversion of motion state.

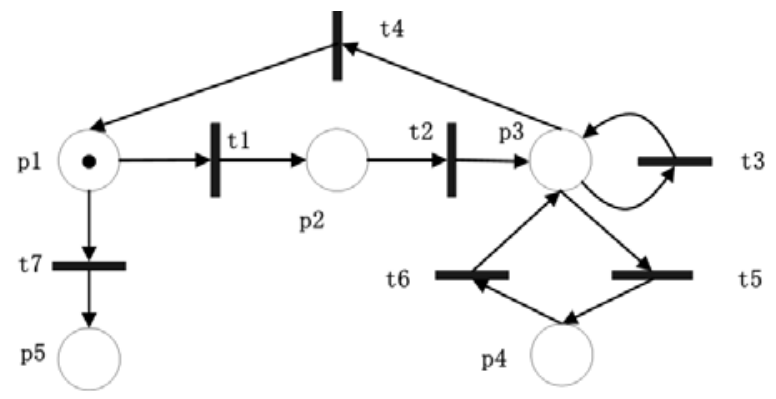

Figure2. Control structure of Petri Net

Action elements initialized (_ Init _), automatically into P1. Change of state action elements realize the recycling process and through the change of command. Because all of the actions and the action based on the situation of sports school, therefore, the design of [4] application of differential effect identical structural elements and technical movement. Six groups of Petrie network elements can be used for $\mathrm{PN}=(\mathrm{P}, \mathrm{T}, \mathrm{F}, \mathrm{K}, \mathrm{W}, \mathrm{M} 0)$, contains elements such as shown in table 1 . 
Table1. Place/Transition instruction for Petri Net

\begin{tabular}{cccc}
\hline Number & Implication & Number & implication \\
\hline $\mathbf{P}_{\mathbf{1}}$ & Initialized states & $\mathrm{t}_{2}$ & PreAction \\
$\mathbf{P}_{\mathbf{2}}$ & Ready condition & $\mathrm{t}_{3}$ & Update \\
$\mathbf{P}_{\mathbf{3}}$ & Running status & $\mathrm{t}_{4}$ & PostAction \\
$\mathbf{P}_{\mathbf{4}}$ & Paused states & $\mathrm{t}_{5}$ & Pause \\
$\mathbf{P}_{\mathbf{5}}$ & Finished states & $\mathrm{t}_{6}$ & Resume \\
$\mathbf{t}_{\mathbf{1}}$ & Start & $\mathrm{t}_{\mathbf{7}}$ & Finish \\
\hline
\end{tabular}

1) $\mathrm{P}=\left\{\mathrm{p}_{1}, \mathrm{p}_{2}, \mathrm{p}_{3}, \mathrm{p}_{4}, \mathrm{p}_{5}\right\}$ defines Place collection of $\mathrm{PN}$.

2) $T=\left\{t_{1}, t_{2}, t_{3} \ldots \ldots t_{7}\right\}$ defines Transition collection of PN.

3) $\mathrm{F} \subseteq(\mathrm{P} \times \mathrm{T}) \mathrm{U}(\mathrm{T} \times \mathrm{P})$ describes the flow in the network, defines the directed arcs between the Place and Transition.

4) $\mathrm{K}: \mathrm{P} \rightarrow \mathrm{N}^{+} \mathrm{U}\{\infty\}$ defines the functional capacity number of Place, $\mathrm{N}^{+}=\{1,2,3 \ldots \ldots\}$.

5) $M_{0}$ defines the initial identification of Petri Net, for every tree in the early state, giving the number of token, general said for the vector form. And there is $\mathrm{M}_{0}=[1,0,0,0,0]^{\mathrm{T}}$.

6) $\mathrm{W}: \mathrm{F} \rightarrow \mathrm{N}^{+}, \mathrm{N}^{+}=\{1,2,3 \ldots \ldots\}$ defines the weight function of arc set

\section{The establishment of the model of sports skilled motion characteristic of digital training system for sports}

Integrity and independence is that exercise library design requirements, we need to achieve the proper classification technique, and in order to achieve the general requirements, the need to ensure that only the action library scalable [5]. The technical classification method and physical motion model based on the task decomposition, we propose a class of athletes in action database structure, down from can be roughly divided into action unit layer, behavior layer and factors of the technical action, as shown in figure 3.

Combined with the human skeleton model analysis and the Petrie network technology design, and in order to convenient and fast construction characteristics of sports action model, it needs through action activity decomposition and classification, to realize the encapsulation of athletes in action. The model database is the key technology for realizing the motion characteristics. As a basic tool generally uniform, mobile library should complete characteristic, independent, and versatile.

Action basic layer: This layer contains only one basic unit. Basic action elements define the basic framework of athletes, describe some of the basic elements, including start, pause, stop action cycle, and the general motion parameters.

Technical action elements layer: Movement of elements from the basic action sports action semantic elements, the most basic feature, but it cannot subdivide the operational semantics, is the most basic of human movement form sports action unit. In the sports elements, usually according to the model and motion of human body joint degrees of freedom.

Motor unit action unit layer: Technology is the many technical elements according to the semantic rules, and certain semantic features, describes the general meaning, semantic features of the technology, but does not contain any action semantics associated to a particular activity.

In the action of basic layer, we design the basic action based on finite state basic class, the class of motion elements of the parent class's behavior and action unit, responsible for the implementation of the technical action of cyclic scheduling; element layer movement, the movement of the basic derivation and package of aircraft motion, attitude adjustment, unarmed, tool operation dynamic operation element cell; cell layer of the movement, according to the semantic features of further action from the action elements and specific action units. 


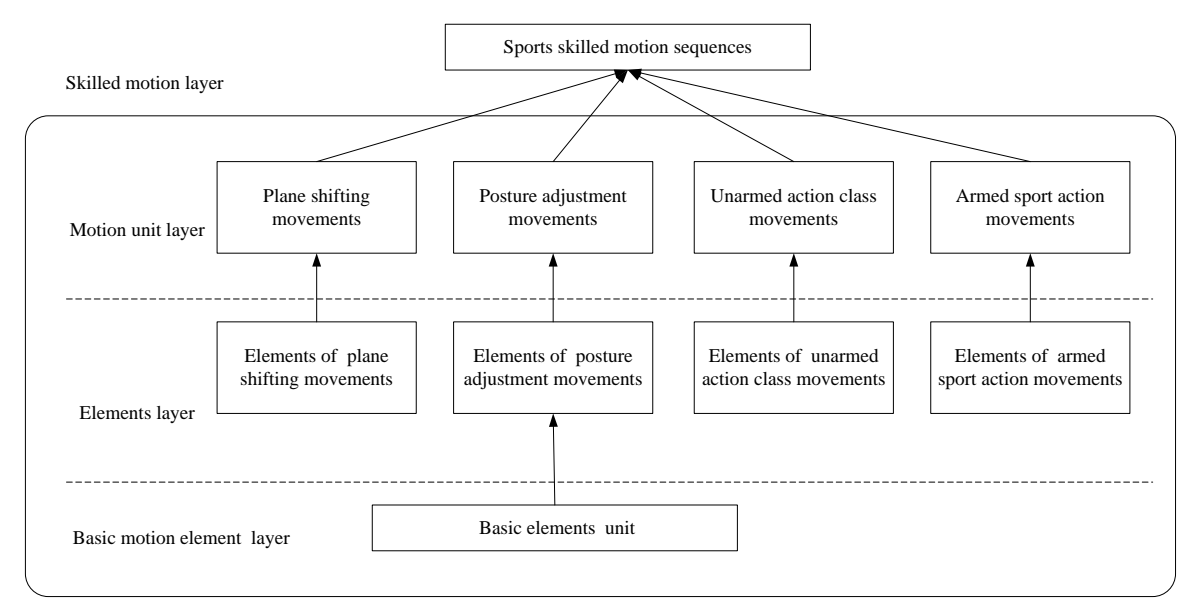

Figure3.The hierarchical model of sports skilled motion characteristic

\section{Conclusion}

In this paper, analysis of the sports connection principle from human skeleton model based on the angle of view, and computer technology in the design of sports technology based on the characteristics of action, combined with the movement skill to set up a model of regular physical activity data. In turn, it found that the association rules between sports skills, research oriented sports digital system to shorten the time effectively training, in order to improve the level of athletes and coaches, provide scientific guidance and important reference.

\section{Reference}

[1] S. Barris, and C. Button: Sports Medicine, Vol.32 (2012) No.12, p.1025

[2] M. Perše, M. Kristan, and J. Perš: Pattern Recognition, Vol.40 (2011) No.4, p.1491

[3] C.G. Lane, R.F. Warren, and F.C. Stanford: Knee Surgery, Sports Traumatology, Arthroscopy, Vol.16 (2009) No.5, p.487

[4] G.Li, L.E. DeFrate, and S.E. Park: The American journal of sports medicine, Vol.35 (2010) No.1, p.102

[5] K. Davids K, C. Button C, and D. Araújo: Adaptive Behavior, Vol.34 (2008) No.1, p.73 